\title{
HOMBRES QUE DECIDEN CUIDAR A PERSONAS ADULTAS DEPENDIENTES EN EL CONTEXTO FAMILIAR. GÉNERO Y PARENTESCO EN TRANSFORMACIÓN
}

MONTSERRAT SORONELLAS-MASDEU DOLORS COMAS D'ARGEMIR NATÀLIA Alonso-ReY Universitat Rovira i Virgili

Resumen: En este artículo analizamos la realidad cotidiana de hombres que, estando todavía en edad laboral, toman la decisión de abandonar sus actividades profesionales para dedicarse al cuidado de sus esposas o de sus hijos adultos dependientes. Se trata de hombres que han priorizado los cuidados y que ejercen de cuidadores en el entorno familiar, combinando los recursos que ponen a su disposición el mercado, el sector público o las iniciativas sociales comunitarias. Entendemos que un suceso inesperado que afecta a la salud plantea lógicas específicas en la forma de afrontar el cuidado y el rol asumido por el cuidador. A partir de una metodología cualitativa, analizamos cómo entienden el cuidado los padres y maridos cuidadores; qué tipo de cuidados realizan; cómo organizan y distribuyen la provisión de cuidados; cuál es la actitud moral con que afrontan los cuidados; y qué conflictos y malestares emergen de la situación de cuidado. Todo ello en el marco de las motivaciones y contexto que abocan a estos hombres a la decisión de cuidar. Lo relevante a nivel teórico es entender que estos hombres que deciden cuidar ponen 
en tensión las prescripciones derivadas del género con las obligaciones generadas por el parentesco.

Palabras Clave: cuidado; hombres cuidadores; cuidado familiar; género; parentesco.

AвSTRACT: In this article, we analyze the daily reality of men who, whilst still of working age, take the decision to abandon their professional activities to dedicate themselves to caring for their dependent wives or dependent adult children. These are men who have prioritized care and who act as caregivers in the family setting, combining the resources made available to them by the market, the public sector or community social initiatives. We understand that an unexpected event that affects health raises specific questions regarding the provision of care and the role of the caregiver. Using a qualitative methodology, we analyze how care is understood by father and husband caregivers; what type of care is provided; how they organize and distribute the provision of care; what is the moral attitude that they adopt towards care; and what conflicts and discomforts arise from the caregiving situation. All of this is examined in the frame of the motivations and context that cause these men to adopt the role of caregiver. At a theoretical level, it is important to understand that these men wo decide to provide care generate tension between gender prescriptions about care provision and the obligations resulting from kinship.

KEYWORDS: care; male caregivers; family care; gender; kinship. 


\section{Introducción ${ }^{1}$}

Las sociedades contemporáneas afrontan los cuidados de las personas adultas dependientes mediante sistemas complejos que combinan diferentes recursos entre los cuales la familia sigue teniendo un papel esencial. La llamada organización social de los cuidados (Daly y Lewis, 2000; Razavi, 2007) pivota sobre cuatro pilares básicos: la familia, el Estado o las políticas públicas, una amplia gama de servicios proporcionados por el sector privado y el ámbito comunitario. La forma en que se combinan las políticas públicas y los recursos privados depende tanto del modelo de estado de bienestar como de la situación económica y organizativa de las familias. Barajando estas variables, las familias se organizan y encajan las piezas que componen su particular mosaico de recursos de cuidado (Soronellas y Comas d'Argemir, 2017). Las demandas de cuidado y la dificultad de proveerlas se han conformado como una «crisis» en Europa (Benería, 2008; Comas d'Argemir, 2014; Pérez Orozco, 2006), y el cuidado familiar ha pasado a ser un elemento central, que se ha visto reforzado por los procesos de deinstitucionalización y refamiliarización que son resultado del impacto de la crisis económica iniciada en el 2008 (y actualmente de la covid-19).

La literatura académica ha constatado que las mujeres son las principales agentes de cuidado, tanto si este se efectúa en la familia de forma remunerada como en los empleos de cuidado; tanto si se trata de personas enfermas o vulnerables como si se trata de personas que puedan valerse por sí mismas. Las mujeres concentran, pues, las prescripciones morales y de género por las que se hallan obligadas a cuidar. Los cambios sociales y culturales, en la familia y en la vida de las mujeres, están acelerando la incorporación de los hombres al cuidado, especialmente en la crianza de los hijos (Alméras, 2000; Meil, 1997; Hanlon, 2012; Abril y otros, 2015), y también, aunque menos, al cuidado de personas adultas depen-

\footnotetext{
I Este artículo se enmarca en dos investigaciones más amplias. La primera es «Hombres cuidadores. Retos y oportunidades para reducir las desigualdades de género y afrontar las nuevas necesidades de cuidados», RecerCaixa, programa impulsado por la Obra Social "la Caixa" con la colaboración de la Asociación Catalana de Universidades Públicas (2014ACUP00045). La segunda es «El compromiso de los hombres con los cuidados de larga duración. Género, generaciones y culturas del cuidado», Proyectos I+D+i del Ministerio de Economía y Competitividad. (FEM2017-83517-R). En ambos casos la IP principal es Dolors Comas d'Argemir.
} 
dientes, muy especialmente maridos ancianos que asisten a sus esposas ancianas y que al estar jubilados no desasisten sus obligaciones laborales (Calasanti y Bowen, 2006; Comas d'Argemir, Alonso y Deusdad, 2018; Milligan y Morbey, 2016; Russell, 2001).

En este artículo nos acercamos a la realidad cotidiana de hombres que, estando todavía en edad laboral, toman la decisión de abandonar sus actividades profesionales para dedicarse al cuidado de sus esposas o de sus hijos adultos en situación de dependencia. Exploramos las lógicas de la incorporación de los hombres al cuidado de sus familiares a partir de la descripción y análisis de casos significativos: maridos que aceptan y normalizan el cuidado; otros que lo sienten como una obligación que provoca malestar; $y$ padres que se abocan al cuidado de hijos enfermos $y$ dependientes desde un cuidado experto y militante que intenta resolver el presente y prever el futuro de la situación.

En artículos anteriores (Soronellas y Comas d'Argemir, 2017; Aguilar, Soronellas y Alonso, 2017; Comas d'Argemir y Soronellas, 2019), hemos abordado la situación de hombres que cuidan de sus esposas o de sus padres/madres en la vejez, situaciones de cuidado que son asumidas como «naturales» dentro de la lógica del curso de vida. En este texto hemos querido omitir los cuidados en la vejez, y tratar específicamente la situación de hombres que, ante el advenimiento de una situación de enfermedad física o mental que no permitirá a las personas afectadas (esposas o hijos) llevar una vida completamente autónoma, han abandonado sus ocupaciones laborales para poder dedicarse al cuidado. Entendemos que la irrupción de un suceso inesperado en la salud dibuja un contexto que puede tener lógicas específicas en cuanto a la forma de afrontar el cuidado y el rol asumido por el cuidador. El reto de este artículo es analizar cómo entienden el cuidado los padres y maridos cuidadores entrevistados; qué tipo de cuidados realizan; cómo organizan y distribuyen la provisión de cuidados; cuál es la actitud moral con que afrontan los cuidados, y qué conflictos y malestares emergen de la situación de cuidado. Todo ello en el marco de las motivaciones y contexto que abocan a estos hombres a la decisión de cuidar. 
Los casos analizados proceden de los datos recogidos en una investigación en curso sobre hombres cuidadores realizada en Cataluña (España). Para esta investigación hemos efectuado 208 entrevistas a hombres que cuidan en el entorno familiar, a hombres que hacen trabajos remunerados de cuidados, y a gestores de servicios de cuidados (públicos y privados), así como a personas receptoras de cuidados, con el objetivo de evaluar las barreras culturales y de oportunidad que inciden en la implicación de los hombres en los trabajos de cuidados, así como los modelos emergentes. La investigación se ha centrado en los cuidados de larga duración de personas adultas con alto grado de dependencia, excluyendo la atención estrictamente sanitaria para centrarnos en el cuidado social (Daly y Lewis 2000). Los seis casos trabajados en este artículo forman parte de las 49 situaciones de cuidado familiar que fueron recogidas durante el trabajo de campo realizado entre 2015 y 2016.

\section{Maridos y padres cuidadores: produciendo género y parentesco}

El hecho de que un suceso inesperado irrumpa en la salud de los familiares más próximos es un factor desencadenante para la implicación de los hombres en el cuidado, lo que redefine los roles y contenidos de género y de parentesco (Banens y Marcellini, 2015). Sin embargo, no es un factor suficiente, ya que ha de ir acompañado de otros aspectos de carácter laboral, social y político que actúan como condicionantes.

Desde el punto de vista laboral, hay que tener en cuenta el papel material y simbólico que tiene el trabajo remunerado en la construcción de la masculinidad. Se ha demostrado que, en el caso de España, así como de los países mediterráneos, el trabajo es un atributo de importancia central para los hombres, especialmente para los de clase obrera, de quienes se considera que depende el sustento de ellos mismos y de sus familias, en tanto que las mujeres tienen el rol de cuidar (Borràs, Moreno, Castelló y Grau, 2012). Hemos de considerar, pues, cuáles son las situaciones que permiten prescindir del trabajo remunerado, rompiendo, así, las prescripciones de género, y priorizar el cuidado de familiares, siguiendo las obligaciones derivadas del parentesco. 
Desde la perspectiva social y política hay que tener en cuenta justamente el rol de las políticas públicas en la provisión de cuidados, pues ello incide en las familias. En el caso de España o de Italia, por ejemplo, con un welfare poco generoso, se exacerba el rol de la familia, y la solidaridad intergeneracional deviene imprescindible (Saraceno, 2010). Esto supone una sobrecarga en las familias en materia de recursos, de gasto económico y de tiempo. Es una especie de solidaridad obligada revestida de moralidad.

Planteamos, pues, que esta priorización del cuidado por parte de los hombres es una elección condicionada, cosa que intentaremos mostrar. Pero lo relevante a nivel teórico es entender que estos hombres que deciden cuidar ponen en tensión las prescripciones derivadas del género con las obligaciones generadas por el parentesco.

El parentesco es, como el género, una construcción social que debe ser tenida en cuenta para entender el modo en que se produce la organización social de los cuidados. Las familias son instituciones de poder que jerarquizan a sus miembros a partir de una estructura según la cual el género, la generación y la posición genealógica se combinan para distribuir las responsabilidades del cuidado de sus miembros. Se producen, pues, intersecciones entre el género y el parentesco que deben ser analizadas como tales (Collier y Yanagisako, 1987). Las mujeres reciben de la sociedad la responsabilidad del cuidado en su condición de mujeres y también por ser madres, esposas e hijas; no obstante, la obligación de cuidar vinculada al rol de parentesco ha sido menos problematizada que el género en la literatura académica (Comas d'Argemir y Soronellas, 2019).

Sostenemos que el parentesco se construye y reproduce a través del ejercicio del cuidado y que las formas de cuidar y ser cuidado configuran el parentesco y estructuran las relaciones entre los parientes (Carsten, 2000; 2004; Borneman, 1997). Cuidar es la expresión más importante del ejercicio del parentesco, a la vez que hacer de pariente es, sin duda, proveer cuidados a los miembros de la familia.

El cuidado puede expresar también la finalización del vínculo y el elemento más significativo de la ruptura entre familiares es la omisión de asistencia, la ausencia de preocupación mutual. Es el caso de las situacio- 
nes de divorcio, donde los excónyuges se desentienden de los cuidados de los exparientes afines (Roigé y Soronellas, 2018). Sahlins (2011) se refiere a la «mutualidad del ser» (mutuality of being) para explicar el vínculo entre las personas emparentadas, a las que define como personas que participan de la existencia de las demás, ejercen la mutualidad, participan de la vida de otros con o sin vínculos biológicos. Carsten (2013) apostilla a Sahlins que también existe una cara amarga en la experiencia del parentesco, que también hay conflicto y que las relaciones pueden ser dañinas y hasta disolverse o desaparecer. Sin duda, el parentesco es un entramado complejo de relaciones y sentimientos, positivos y negativos, que pueden ser visibilizados perfectamente en las situaciones de cuidado y muy especialmente en las situaciones de atención familiar a las personas adultas, enfermas o ancianas, puesto que son percibidas culturalmente como una carga (Manceron y Segalen, 2012) y pueden generar contradicciones y conflictos entre quienes se sienten obligados a llevarla.

Los hombres que cuidan priorizan el vínculo del parentesco por encima de los dictados del género, algo que les permite no cuestionar demasiado su masculinidad (Comas d'Argemir y Soronellas, 2019). Son hombres que, hasta un cierto punto, para asumir las responsabilidades de cuidado (como veremos en el análisis de los casos) cruzan las barreras del género y transgreden la masculinidad hegemónica care free (Connell, 1995; Connell y Messerschmidt, 2005) y los modelos de cuidado en los que fueron socializados. Los hombres expresan desde la conyugalidad y la paternidad la obligación moral que les compromete a cuidar de sus esposas e hijos. El sentido de esta obligación reside en la concepción del cuidado como un don que genera deuda social (Comas d’Argemir, 2017), que crea vínculo social y que circula recíprocamente entre generaciones y entre cónyuges. Como señalan Comas d'Argemir y Chirinos (2017), se han producido cambios en el sentido de la reciprocidad del cuidado. El mismo hecho de que implique a los hombres ya es una novedad, pero también se ha transformado el sentido de la circulación intergeneracional del cuidado, que ha dejado de ser circular (los padres cuidan a los hijos y estos devuelven el cuidado a los padres en la vejez) para pasar a ser lineal (los padres cuidan a los hijos y estos devuelven formas de gestión indirecta del cuida- 
do). Respecto al cuidado conyugal, los hombres en su rol de breadwiners, se consideraban liberados de proveerlo, y ante una situación de necesidad de atención, las hijas o las nueras eran las responsables del cuidado siguiendo el modelo de reciprocidad circular mencionado. Los datos de nuestra investigación muestran que actualmente los maridos se comprometen con el cuidado de sus esposas, un cuidado que ellos entienden en el contexto de la devolución de los dones de cuidado que han recibido de ellas a lo largo de la vida.

Los compromisos de cuidado de los maridos y padres que cuidan son asumidos desde la moral del parentesco, pero, al mismo tiempo son negociados tomando en cuenta no tan solo el rol de género y parental, sino también atendiendo a las condiciones sociales y económicas y a la disponibilidad de recursos de atención (Finch, 1989). Los roles de parentesco se negocian y se transforman, modificando, si es conveniente, el sentido de obligación y de reciprocidad que se les atribuye. Debemos tener en cuenta, además, que las transformaciones de la familia y de la sociedad han contribuido a hacer más electivas las obligaciones del parentesco, pero sus bases morales, aunque transformadas, siguen vigentes.

A continuación, presentamos seis casos de maridos y padres que han asumido el cuidado de esposas e hijos en situaciones inesperadas e indeseadas. El compromiso adquirido es expresado desde la obligación moral, desde la reciprocidad, desde los afectos y la recompensa emocional, pero también desde la contradicción, el malestar y el conflicto. Los casos nos permiten comprender por qué se toma la decisión de dejar el trabajo remunerado para atender los cuidado, cómo se asume y organiza la situación, compartiendo con otros familiares, con la red social y comunitaria, o con personas remuneradas. Nos interesa también describir con qué actitud se afronta la interdependencia con la persona cuidada, desde la normalidad y el consentimiento o desde el conflicto y el malestar. Y, por último, ¿qué significa cuidar para cada uno de ellos? Es interesante observar si el cuidado se entiende como algo complejo y holístico, que tiene que ver con la gestión y el mantenimiento de la vida atendiendo al bienestar físico y emocional de las personas (Pérez-Orozco y López-Gil, 2011), o si los cuidados se conciben desde el conocimiento experto y la ad- 
ministración de atenciones más centradas en la gestión de las dolencias o, incluso, en la previsión del futuro de la persona cuidada.

\section{Maridos cuidadores de esposas enfermas}

\subsection{Mikel: «Decidimos que mejor que yo no la iba a cuidar nadie»}

Mikel tiene 53 años y su mujer, Menchu, tiene una enfermedad rara que comenzó a manifestarse en 1993, cuando ya tenían una hija. Dos años después estaba en una silla de ruedas y cinco años más tarde había derivado en una tetraplejia. Residían en el País Vasco, y en 2002 decidieron trasladarse a Cataluña, donde pudieron construir su casa adaptada. Mikel decidió dejar su trabajo para cuidar a su esposa.

Menchu obtuvo una pensión de gran invalidez, lo que les aseguraba no tener problemas económicos. Tuvieron durante un tiempo una mujer contratada durante algunas horas al día, pero, según Mikel: «Haciendo números, me salía más a cuenta casi quedarme en casa que ir a trabajar». Según Mikel, la percepción de la pensión les ha dificultado acceder a otros apoyos públicos y tan solo ha conseguido ayudas para eliminar las barreras arquitectónicas de su domicilio.

A pesar de que Mikel afirma que a su mujer le costaría tener a alguien desconocido en la casa, señala que sería bueno para él poder contar con alguien durante unas horas que le permitiera tener tiempo libre. Su hija vive en la ciudad de Tarragona con su marido y su hijo y le ayuda ocasionalmente, pero Mikel expresa no querer «cargar a [su] hija con los problemas de [su] mujer», al tiempo que enfatiza que a su esposa cada vez le cuesta más estar sola o estar sin él. Esto tiene implicaciones para sus propias necesidades de cuidado: no es posible enfermarse, según sus palabras, ya que tendría consecuencias en la atención a su esposa. Es por ello que en su proyección de futuro imagina buscar una residencia donde puedan vivir juntos.

Cuidar es para Mikel «estar con ella», lo que incluye tanto las actividades instrumentales como las más relacionales. Esta situación no está exenta de complejidades porque estar todo el día juntos no es fácil y «lo bonito», según dice, sería estar todo el tiempo hablando, pero el cuidado 
requiere muchas otras cosas. Mikel indica que el hecho de que su mujer tenga una dependencia física pero no cognitiva significa que «ella es quien dirige» y él, quien ejecuta, lo cual implica negociaciones e incluso tensiones.

\subsection{Darío: «Prefiero tener el estrés yo que no tenerla a ella»}

Dos años y medio atrás, Mónica, la esposa de Darío, de 43 años, fue diagnosticada con una enfermedad autoinmune grave. Según el relato de ambos, los primeros indicios de la enfermedad se remontan probablemente al momento posterior al nacimiento de su hija pequeña (tienen dos), cuando le diagnosticaron una depresión posparto. El proceso de aparición y diagnóstico de esta enfermedad degenerativa es narrado como algo largo y angustiante. Las primeras manifestaciones aparecieron de forma repentina y resultaban muy invalidantes. La imprevisibilidad y la dificultad de poder ejecutar tareas cotidianas son remarcadas por Darío como dos cuestiones fundamentales para comprender por qué y cómo desempeña su papel de cuidador.

A raíz de la enfermedad de Mónica, Darío decidió dejar el empleo a media jornada que tenía, pues explica que era imposible continuar trabajando y, además, asumir el cuidado de su esposa y de los hijos. En el momento de la entrevista, desempeñaba algunas actividades de forma desregulada para obtener ingresos y, remarcando su papel activo en las gestiones administrativas, había tramitado una pensión de invalidez para Mónica. No ha logrado obtener prestación económica alguna ni acceder a un servicio de atención domiciliaria. Por todo ello, la situación económica de ambos se ha visto profundamente afectada.

La relación con familiares y amistades a raíz de la enfermedad se ha visto deteriorada. En el caso de los primeros, la pareja señala un alto grado de incomprensión de la enfermedad de Mónica, además de que geográficamente se encuentran distantes. Respecto de los segundos, como sucede en varios casos similares, los amigos van desapareciendo. Si bien el matrimonio recurre a una iglesia, no la señalan como un apoyo, más allá de participar en algunas de las actividades. Algo similar sucede con asociaciones de personas afectadas por la misma enfermedad que Mónica. 
Mónica puede en ocasiones ejecutar ciertas tareas de cuidados en relación con sus hijos, pero no puede programarse que sea ella quien las desempeñe. Sin embargo, dado que su capacidad cognitiva no está afectada, Darío afirma que es ella quien planifica y él, quien ejecuta. Darío describe en su relato cómo desde un primer momento de confusión por el advenimiento de una situación grave e inesperada su vida cotidiana y su rol en la familia se fueron transformando para convertirse en «las piernas» de su mujer, como él describe. Pero esta transformación es narrada sin cuestionamientos, como una respuesta natural a la excepcionalidad de las circunstancias: «Somos una familia, somos un paquete». Señala, no obstante, las dificultades derivadas de la falta de recursos a que se enfrentan, especialmente desde los servicios públicos. Su principal demanda al respecto sería poder contar con una persona - una mujer, según señala- que pudiera cuidar a su mujer de forma remunerada, brindándole tiempo a él, y, también porque, según afirma, una mujer puede llegar a donde él no llega. Cuidar para él es «estar pendiente de todo», atender a su mujer es, nos dice, «como tener otro niño».

\subsection{Miguel: «El cuidador es vampirizado o comido por la persona que cuida»}

Miguel tiene 63 años, está jubilado y vive con Antonia, su esposa, quien sufrió un ictus que le dejó con un 83\% de minusvalía. Mantenían una división de tareas tradicional: su esposa se dedicaba al cuidado de la familia mientras él trabajaba largas jornadas fuera de casa. Miguel tuvo que adelantar sus previsiones de jubilación y dedicarse a cuidar de su esposa.

Antes de sufrir el ictus, Antonia se desplazaba periódicamente a Andalucía para cuidar a su madre. Este cuidado intergeneracional no se produce en el caso de las hijas de Miguel; ambas tienen sus empleos e hijos y una de ellas vive lejos. Han contribuido a readaptar la vivienda de sus padres y les visitan ocasionalmente, pero no son un gran apoyo para Miguel. Él lamenta la pérdida de libertad, el fracaso de los proyectos de futuro y el hecho de tener una relación tensa con su esposa y sus hijas. Se siente incomprendido y no cuenta con un grupo de amistades con quien poder compartir su situación. Lo expresa claramente cuando señala que 
el cuidado «vampiriza», una expresión que ilustra la sensación de ahogo que experimenta.

Recibe apoyo diario de una trabajadora familiar y de una trabajadora del hogar contratada. Su esposa es atendida también en un centro de día de una fundación privada algunos días por semana. Esto permite a Miguel disponer de tiempo libre, «escapar», aunque lo utiliza también para visitar a su propia madre, de 96 años, a quien cuida la hermana de Miguel. Las tardes y los fines de semana son particularmente difíciles puesto que debe asumir solo y sin relevo las tareas de cuidado. Para Miguel su esposa «no es la de antes, es otra persona», y si bien las tensiones conyugales eran previas a la enfermedad, se han ido acentuando. Lo que le ha llevado a pensar en separarse. Ante esta debilidad del vínculo en términos afectivos y de planes conjuntos, lo que queda es un sentido de obligación hacia la madre de sus hijas, según él mismo dice. Este sentido de obligación, arraigado en el sistema de valores en el cual fue educado, es el que le motiva a seguir cuidando de su esposa, no sin dejar de expresar el conflicto que le supone no poder disfrutar de su propia vida.

\subsection{Entre la naturalización y los malestares}

Como hemos señalado, en los tres casos, las situaciones de dependencia se manifestaron de manera imprevista en parejas que aún se encontraban en edades activas o cercanas a la jubilación. La vinculación con el mundo laboral y la situación socioeconómica son esenciales para comprender la manera de estructurar, gestionar y actuar de estos maridos cuidadores en cuanto a la situación de dependencia. Cuidar necesita tiempo y recursos, por lo que quienes se encontraban trabajando debieron anticipar sus retiros y gestionar con dificultad los recursos económicos y servicios procedentes de un sistema de protección social que resulta escaso.

Si bien en todos los casos la situación de cuidados aparece de manera inesperada, es vivida de manera divergente. En el primero, el marido se hace cargo de los cuidados, asumiendo que es la mejor opción posible, y, aunque no está exenta de conflicto, aparece como una situación naturalizada. Darío, con una situación económica difícil, ha de compaginar el cuidado con algunos trabajos puntuales y ayuda social. Mikel señala que 
negocia los cuidados con su esposa, la cual sigue organizando, a pesar de su escasa autonomía física, las actividades diarias, cosa que ocurre también en el caso de Darío.

La situación de Miguel es bien diferente de las de los demás, puesto que su esposa sufre fuertes deterioros psíquicos que transforman el vínculo entre ellos, si bien ha señalado en la entrevista tensiones previas a la manifestación de la enfermedad. El cambio ha provocado incluso extrañamiento hacia su esposa: «Es otra persona», nos comentaba. También ha enfatizado la alteración de sus planes de jubilación, una etapa en que pensaban dedicarse a «disfrutar de la vida», cosa que contrasta con su situación actual.

La dependencia altera los roles conyugales en los tres casos. Los hombres habían desempeñado el rol de proveedores y las mujeres se dedicaban a los trabajos domésticos (incluso si ellas tenían un trabajo remunerado). En el presente, los hombres pasan a asumir tareas de cuidados que no habían ejecutado hasta entonces. Las motivaciones para cuidar derivan de un énfasis en el vínculo matrimonial y en las obligaciones de cuidado que se entienden derivadas de dicho vínculo. Existe un sentido de reciprocidad en tanto que se apela al rol de la mujer como cuidadora de otros (la madre de los hijos), lo que da sentido a que los maridos devuelvan los cuidados aun cuando no era algo que se esperase de ellos. Aquí es importante destacar que se produce una inversión de los roles de género a consecuencia de la intersección de las categorías de género y las de parentesco: ser esposo obliga al cuidado (Comas d'Argemir y Soronellas, 2019). En dos de los casos las parejas tienen hijas adultas, y si bien participan en los cuidados, esta participación es minimizada o incluso rechazada por parte de los maridos (padres), que entienden que son ellos quienes deben asumir los cuidados, una afirmación del rol cuidador que bascula entre la reivindicación de la propia agencia y el reconocimiento de un cambio generacional que libera a los hijos del cuidado de los padres (Conlon et alii, 2016). Los apoyos recibidos en todos los casos resultan insuficientes y la vinculación al sistema público de prestaciones es descrita como bastante inalcanzable y lejos de resolver las necesidades de cuidado. El deseo de tener tiempo para sí mismos es una constante en los relatos. 
Mediante los casos de Mikel, Darío y Miguel, hemos querido acercarnos a tres experiencias distintas de maridos cuidadores, las cuales ilustran, por un lado, que el cuidado puede ser aceptado y naturalizado ante la aparición de una enfermedad sobrevenida y que, a pesar de ello, puede también provocar angustias y malestares ocasionados por la percepción de que se ha truncado de repente el curso esperado de la propia vida. En todos ellos, es el rol de los hombres en un sistema de género y parentesco determinado el que nos ayuda a comprender sus motivaciones para cuidar, así como las transformaciones y tensiones de sus propias experiencias como maridos cuidadores. Veamos a continuación qué ocurre con los padres que cuidan de sus hijos adultos dependientes.

\section{Padres cuidadores de hijos enfermos o discapacitados}

\subsection{Jordi, cuidador y activista: «Ha sido una historia de lucha»}

Jordi tiene 66 años. Está casado con Inma y tienen un solo hijo, Arnau (40 años), a quien diagnosticaron esquizofrenia cuando tenía 26 años, después de una década de problemas debidos al consumo de alcohol y otras drogas. Actualmente Jordi está jubilado. Tenía una pequeña empresa de publicidad en la que trabajaba también su esposa. Los tres comparten actualmente el mismo domicilio, en un barrio obrero de Barcelona. Actualmente, Arnau tiene la enfermedad estabilizada, pero no puede tener un trabajo, ya que no consigue concentrarse para realizar tareas concretas. El objetivo de los padres es que consiga tener autonomía.

Cuando Arnau empezó a sufrir brotes psicóticos con episodios de violencia, tuvieron que internarlo en un psiquiátrico varios meses. El joven tomó conciencia de su enfermedad cuando lo juzgaron por una agresión. Era el año 2008, y a partir de entonces cambiaron los hábitos de consumo, aceptó medicarse y vivir en la casa familiar, e inició actividades educativas. «Tiene una vida ordenada», dice su padre, pero solitaria, sin amigos, dependiente de los padres.

Jordi describe la situación como una historia de lucha. «Estamos enfermos también». La preocupación, el insomnio y los sentimientos de culpa aparecen constantemente, y tanto él como su esposa tuvieron que 
ir al psicólogo. «La carga moral es muy fuerte». Jordi e Inma se distribuyen el cuidado de su hijo. Jordi le acompaña a actividades y espacios masculinos, como el fútbol o el café. También vigila que tome la medicación. Y, sobre todo, se dedica al activismo social, procurando dar a conocer los problemas laborales y sociales que sufren las personas con trastornos mentales. La esposa, por su parte, hace buena parte de las tareas domésticas y tiene una actitud más protectora respecto al hijo, con quien se comunica mejor. "Cuidar es educar», dice Jordi. Considera que las indicaciones que se producen en el día a día contribuyen a educar el comportamiento del hijo enfermo, que compara con un niño a quien hay que orientar en todo. Esta es una tarea constante, solapada, intensa. E insiste en que él mismo ha tenido que aprender a tener paciencia, a no irritarse y a escuchar a su hijo.

Con la enfermedad de Arnau, se produce un repliegue del núcleo familiar. Jordi e Inma van perdiendo la relación con amigos y familiares, no solo debido a la actitud de los demás, sino también a la de ellos mismos. «Los que estamos afectados dejamos a los demás», porque, ante la gravedad del problema que se padece, todo parece superfluo e irrelevante y porque cansa la incomprensión de la enfermedad mental y el tener que explicarse constantemente. Por esto el activismo social da sentido a la vida de Jordi y le proporciona, además, un nuevo marco de amistades y relaciones con personas que comparten unos mismos problemas, con las que pueden entenderse más fácilmente e incluso introducir el humor y la ironía en su relación.

\subsection{Frederic, experto y disciplinado: «Él está voluntariamente obligado»}

Frederic era un empresario que se jubiló para poder atender mejor a su hijo, afectado por un trastorno mental. Actualmente tiene 66 años y comparte el domicilio en Barcelona con su esposa y con Adrià, su hijo menor, de 32 años. Tiene otro hijo, José, casado y que tiene su vida independiente.

La enfermedad de Adrià se manifestó cuando el joven tenía 25 años y Frederic considera que el consumo abusivo de drogas es la causa del brote psicótico que sufrió. El joven abandonó los estudios y desde enton- 
ces no ha podido afrontar la vida por sí solo. Cuando su salud empeoró lo tuvieron que ingresar en un hospital psiquiátrico. Al igual que en el caso de Jordi, Frederic tiene un fuerte sentido de culpa del que intenta desprenderse. «¿Por qué tuvo que consumir drogas?» es su pregunta. Su hijo mayor, José, le culpa, además, de tener una actitud condescendiente con Adrià, al que le permite poder vivir sin trabajar y seguir siendo una persona inmadura.

Ante la enfermedad de su hijo, Frederic sintió la necesidad de informarse y de convertirse en experto. Se jubiló para poder asistir a congresos, a jornadas y debates, para leer e informarse respecto a los trastornos mentales, y para participar en el movimiento asociativo. «Si queremos cuidar a esta persona nos hemos de informar y formar para poder acompañarlo correctamente», nos dice. Esta actitud racional, basada en el intelecto y el conocimiento, dista, en cambio, de la emocional. Frederic apenas se comunica con su hijo, quien cuando está en la casa se encierra en su habitación y se refugia en Internet. El joven, sin embargo, sí hace vida social con su grupo de amigos.

Cuando Adrià salió de la clínica psiquiátrica, sus padres le propusieron firmar un pacto de convivencia. En el pacto se definen los horarios y actividades que realizar, la obligación de seguir el tratamiento médico y el abandono del consumo de drogas. Y se especifica al final que, si no se cumple lo acordado, no podrá seguir viviendo con ellos. Hacen, así, un planteamiento radical (de caixa o faixa, según la expresión en catalán). La actitud del padre (la madre es más indulgente y protectora) es clara y concisa: «O haces esto, o no puedes estar con nosotros».

El tratamiento, que es uno de los puntos esenciales de este pacto, no solo incluye aspectos médicos, sino también una disciplina que permita al joven incorporarse a la vida social y laboral. En el momento de la entrevista, Adrià acudía diariamente a un hospital de día y, según Frederic, lo hacía «voluntariamente obligado». La fase siguiente del tratamiento sería la formación para conseguir la inserción laboral, a la que Adrià se resiste, pues no soporta la rigidez de horarios y tareas. «Es la última gran batalla que tenemos como familiares». 
Frederic participa de la vida asociativa para orientar a las familias que se encuentran en situaciones similares y sienten temor por el futuro de sus hijos. No hay que esperar, hay que actuar. «El día de mañana es hoy. Cuando nosotros no estemos ya será tarde». Por esto Frederic defiende que su papel como cuidador es conseguir la autonomía del hijo hoy enfermo y el principal recurso es el intelectual, tener información y formación.

\subsection{Plácido, resignado y obligado: «He hecho el papel de padre y de madre»}

Plácido tiene 66 años y se jubiló anticipadamente a los 60 años cuando su hijo pasó a vivir con él. Emigrante llegado desde Galicia, trabajó como mecánico industrial y vive en una población obrera. Cuida de su hijo (Darío), de 37 años, afectado de un trastorno bipolar, y quien, en el momento de la entrevista, estaba ingresado en un centro para drogodependientes. El matrimonio de Plácido duró pocos años, la esposa tenía la custodia del hijo y él lo veía periódicamente. Señala que detectó tarde la enfermedad de su hijo porque su madre se lo ocultó, aunque él notaba que algo no iba bien, especialmente cuando empezaron los brotes de violencia. Se ocupó del hijo facilitando que trabajara en la misma empresa que él, pero duró poco menos de un año. También fue breve la relación de pareja que tuvo Darío y cuando se separaron el joven pasó a vivir con su padre.

Plácido hacía la limpieza del hogar, se encargaba de la ropa y de cocinar y también se ocupaba de controlar la medicación. Nunca consiguió que su hijo hiciera una vida ordenada y a Plácido le desconcertaban los cambios de carácter, el consumo de drogas, el caos de su habitación y de su vida, y hubo incluso un intento de suicidio. Se inició el periplo por los hospitales psiquiátricos con internamientos esporádicos. El relato de Plácido está colmado de episodios conflictivos con su hijo: destrozos en la casa, insultos y agresiones. La violencia fue subiendo en intensidad, hasta el punto que denunció a su hijo varias veces y finalmente los jueces decretaron una orden de alejamiento. Ante esta situación extrema, su madre aceptó que volviera a vivir con ella.

Fue entonces cuando Darío tomó conciencia de su enfermedad y aceptó que le ingresasen en un centro para drogodependientes, que pro- 
porcionaba tratamiento para él y terapia y actividades para los padres. Plácido y su exesposa asumieron afrontar esta nueva etapa recuperando una mejor relación entre ellos y los fines de semana se combinan para acogerlo en los hogares respectivos.

La vida social de Plácido se ha reducido notablemente y se centra ahora en la participación en las actividades de la asociación (no como activista, sino como usuario) y en el cuidado del hijo. Confiesa que le ha costado asumir este papel de cuidador. "Soy hombre y tengo el toque machista que corresponde a la educación que recibí», nos dice. «Mi refugio era el trabajo». Considera que las mujeres tienen «instinto maternal» y por ello el cuidado de los hijos les corresponde a ellas. Si los hombres cuidan, es porque no tienen otro remedio, porque la situación obliga. Este ha sido su caso. «He hecho el papel de padre y de madre», pero insiste en que le ha costado un esfuerzo adicional hacerlo por el hecho de ser hombre. Y una conclusión final: a pesar de los conflictos y de la violencia, y a pesar de que Plácido llegara a denunciar a su hijo y a echarlo de casa, Darío es su hijo, y esto obliga a que tenga una responsabilidad sobre él. El afecto no aparece en la entrevista en ningún momento; la obligación, sí. Tampoco aparecen la rabia, la culpa o el sentido de injusticia, sino la resignación.

\subsection{Padres que comparten el cuidado o que sustituyen a la madre}

Los padres que hemos ejemplificado tienen prácticamente la misma edad, que supera los 60 años. Los tres adelantan su edad de jubilación para ocuparse de sus hijos, complementando el cuidado de sus esposas o bien sustituyéndolas. Son hombres que han sido educados en una normativa de género que diferencia claramente los roles de los hombres y de las mujeres. La enfermedad que sufren los hijos desborda la capacidad de las madres para cuidarlos y provoca la implicación de los padres. Al tratarse de hijos con trastornos mentales, la crisis o los brotes de violencia interpelan especialmente a los hombres, que inicialmente pueden responder también de forma violenta para pasar a ejercer las funciones de contención y control. El sexo de la persona cuidada es importante, pues el padre se siente más capaz de acompañar al hijo varón en actividades masculinas, sea proporcionándole trabajo, sea asistiendo a partidos de 
fútbol o compartiendo actividades de ocio. En dos de los casos, el hombre complementa así las responsabilidades de la esposa y la distribución de roles en la familia se adapta a la normativa de género tradicional, aunque esto se concreta desde grados distintos de implicación. En cambio, Plácido sustituye a su esposa, al estar separados y vivir en localidades distintas. Jordi y Frederic, por su parte, se implican fuertemente en el cuidado de su hijo desde la representación pública, al liderar asociaciones que trabajan para reivindicar derechos o para ayudar a familias que se encuentran en situaciones semejantes. En todos los casos el cuidado redefine sus vidas de forma totalizadora.

Constatamos, pues, que los padres cuidadores «hacen género» desde su condición de hombres, adaptando los roles tradicionales a su particular situación e implicación en el cuidado. Subrayamos también que «hacen parentesco» desde su rol de padre, por el que se responsabilizan del hijo enfermo, aunque, como hemos comprobado, ejercen la paternidad con intensidades y contenidos diferenciados (Comas d'Argemir y Soronellas, 2019).

En las situaciones que hemos comentado se realiza una distinción clara entre el cuidado y las tareas domésticas. No existe una formulación integral del cuidado que incluya las dos vertientes, sino que se restringe el concepto de cuidar a resolver la necesidad específica de la persona enferma, pudiéndose ampliar en función de la evolución de la enfermedad o la discapacidad. Si la situación requiere tener que alimentar, por ejemplo, esta actividad se incorpora al concepto de cuidado, pero no necesariamente el cocinar o hacer la compra, consideradas tareas domésticas. La presencia de la esposa separa todavía más estas funciones.

El apoyo familiar y social es variable y se complementa con el marco asociativo y con el uso de servicios sociales o sanitarios. Observamos en todo caso un repliegue en el hogar y en el núcleo de relaciones de mayor proximidad. Todos relatan en mayor o menor medida la disminución de sus relaciones sociales y el distanciamiento de amigos y familiares porque la situación de cuidado absorbe su tiempo y también sus mentes y porque les agota explicar sus dificultades y escasos avances. Las asociaciones, o los grupos de ayuda mutua, permiten, en cambio, compartir experiencias 
similares y generan nuevas formas de relación y de amistad. Es así que el cuidado cambia sus vidas, sus relaciones, y también sus perspectivas de futuro respecto a ellos mismos y a sus hijos.

\section{Conclusiones}

Ser padre o marido de hijos adultos y esposas a quienes ha sobrevenido una situación de dependencia motiva a los hombres a cuidar abandonando la actividad profesional, de acuerdo con los condicionantes que modelan esta decisión. Así como los dictados del género liberaron a estos hombres de las tareas del cuidado, en la actualidad, estos padres y maridos ejercen su rol cuidador y lo hacen desde un sentido de obligación moral que les impele a proveer atenciones cuando las circunstancias excepcionales lo demandan. La excepcionalidad es un factor esencial que tener en cuenta, puesto que en todos los casos de los que nos hemos ocupado se trata de hombres que habían estado plenamente dedicados a sus actividades laborales, mientras que las responsabilidades del cuidado eran de sus esposas. Observamos diferencias según los recursos económicos disponibles para poder subsistir. Mientras que en la mayor parte de situaciones analizadas los hombres han dejado su trabajo anticipando su edad de jubilación, en el caso de Darío, el más joven, la precariedad laboral incentiva dejar el trabajo y vivir de la prestación económica y ayudas sociales. En todos los casos, se dispone de recursos económicos para sobrevivir, pero no suficientes para externalizar el cuidado en terceras personas. Solo Miguel tiene apoyo externo (trabajadora familiar y empleada de hogar).

Todos ellos expresan sentirse moralmente obligados a cuidar de sus familiares; el vínculo de parentesco les compromete al cuidado. Los maridos, por razón del vínculo conyugal y la fidelidad hacia unas esposas con las que se ha compartido la vida y de las que se han recibido dones de cuidado. La obligación moral para con sus esposas puede expresarse, bien desde el malestar y el disgusto por considerar que es la única solución posible, bien desde la aceptación racionalizada del cuidado conyugal como la mejor opción. En cuanto a los padres, afrontan el cuidado de sus hijos adultos asumiendo la responsabilidad de la relación de filiación que les 
une con ellos, con mayor compromiso que cuando eran niños. Asumen junto con sus esposas el cuidado de sus hijos dependientes, aun cuando se produce una segregación de las tareas que afronta cada uno de los progenitores, siendo los maridos los responsables de planificar la situación, de gestionar con la Administración el reconocimiento de derechos y la provisión de ayudas y un cierto mantenimiento de la vida social y pública de sus hijos.

Los hombres cuidadores, padres y esposos, enfrentan la organización de los cuidados con ayudas más o menos esporádicas de familiares, mayormente mujeres (suegras, hijas), y, más a menudo, con el trabajo de personas de un servicio público de ayuda en domicilio (SAD) o de mujeres contratadas para atender el trabajo doméstico y, eventualmente, colaborar en los cuidados. Alrededor de estos hombres cuidadores se estructura un conjunto fragmentado de recursos que configura un «mosaico de cuidados» en el que los hombres participan, en parte, realizando cuidado directo, y, en otra parte, gestionando el correcto encaje de estos recursos. La gestión y la planificación toman una dimensión muy importante en el relato de estos hombres cuidadores puesto que entienden la organización cotidiana y, especialmente, la anticipación de las necesidades futuras de las personas cuidadas como parte esencial de su labor cuidadora. La planificación, la movilización social, la realización de trámites con la Administración y la contratación de servicios están muy presentes en sus testimonios como componentes significativos del conjunto de tareas que se necesitan para atender las necesidades del cuidado de sus esposas e hijos, y, en algunos relatos, aparecen como actividades que prolongan las realizadas durante la vida profesional activa de padres y maridos cuidadores entrevistados.

Nos preguntábamos también cómo entienden el cuidado los hombres participantes de nuestra investigación. En algunos casos, especialmente los padres que cuidan de sus hijos conjuntamente con sus esposas, entienden el cuidado desde las acciones específicas y, en cierto modo, especializadas. Por ejemplo: la realización de las curas recomendadas por el personal sanitario, el acompañamiento en la realización de actividades físicas (deporte) o sociales (sociabilidad), y el control de las visitas médi- 
cas. Ello no quiere decir que no contemplen también las tareas domésticas básicas, como la limpieza y la alimentación diaria, como acciones de cuidado de sus hijos, pero no las mencionan prácticamente en las entrevistas porque es una parte del cuidado que realizan sus esposas, algo que ellos naturalizan. Los dictados del género están más presentes en la distribución de roles de cuidado entre los padres/madres que cuidan hijos adultos. En cambio, los esposos tienen una visión más holística del cuidado, en el sentido de que deben atender en solitario (aunque con el mosaico de recursos al que aludíamos antes) a todas las necesidades que sus esposas no pueden atender por sí mismas. Hemos visto en los casos analizados que, cuando es cognitivamente posible, son las mismas esposas quienes «dirigen» o acompañan a sus maridos en la actividad del cuidado, aportando las indicaciones necesarias.

Los casos de hombres cuidadores analizados evidencian que parentesco y género están en proceso de transformación en el contexto de los cambios sociales y culturales que han derivado en la crisis de los cuidados. Se trata de padres y maridos que han incorporado el cuidado como algo propio y que han empezado a traspasar las barreras impuestas por las categorías de género y parentesco que, en su condición de hombres, padres y maridos, les habían liberado de la obligación de cuidar. En sus maneras de cuidar hallamos continuidades de los modelos en los que fueron socializados, pero también rupturas, grietas desde las cuales asumen, a menudo en solitario, el compromiso de cuidar.

\section{Bibliografía}

Abril, P., Amigot, P., Botía-Morillas, C., Domínguez-Folgueras, M. J., Jurado-Guerrero, T., Lapuerta, I., Martín-García, T., Monferrer, J. y Seiz, M. (2015). «Ideales igualitarios y planes tradicionales: análisis de parejas primerizas en España». Revista Española de Investigaciones Sociológicas (REIS), 150, 3-21.

Aguilar, C., Soronellas, M. y Alonso, N. (2017). «El cuidado desde el género y el parentesco. Maridos e hijos cuidadores de adultos dependientes». E-Quaderns, 22 (2), 82-98. 
Alméras, D. (2000). «Procesos de cambio en la visión masculina de las responsabilidades familiares». En J. Olavarría y R. Parrini (eds.). Masculinidad/es, Identidad, sexualidad y familia. Santiago: FLACSO-Chile.

Banens, M. y Marcellini, A. (2015). «Ces hommes qui prennen soin d'autri. Étude de quelques figures masculines de l'aide intrafamiliares». ALTER, European Journal of Disability Research, 9, 195-206.

BENERÍA, L. (2008). «The crisis of care: International migration and public policy». Feminist Economics, 14 (3), 1-21.

Borneman, J. (1997). Cuidar y ser cuidado: El desplazamiento del matrimonio, el parentesco, el género y la sexualidad. Revista Internacional de Ciencias Sociales, 54.

Borràs, V., Moreno, S., Castelló, L. y Grau, A. (2012). «Male hegemony in decline? Reflections on the Spanish case». Men and Masculinities, 15, 406-423.

Calasanti, T. y Bowen, M. E. (2006). «Spousal caregiving and crossing gender boundaries: Maintaining gendered identities» Journal of Aging Studies, 20, 253-263.

Carrasco, C., Borderías, C. y Torns, T. (2011). «Introduccion: El trabajo de cuidados: Antecedentes históricos y debates actuales». En C. CArrasCO, C. Borderías y T. Torns (eds.). El trabajo de cuidados: Historia, teoría y políticas. Madrid: La Catarata.

CARsten, J. (2000). «Introduction: Cultures of relatedness». En J. CARSTEN (ed.). Cultures of relatedness: New approaches to the study of kinship. Cambridge: Cambridge University Press.

- (2004). After kinship. Cambridge, England: Cambridge University Press. -(2011). Substance and relationality: Blood in contexts. The Annual Review of Anthropology, 40, 19-35.

- (2013). What kinship does and how. Journal of Etnographic Theory, 3 (2), 245-251.

Collier, J. F. y Yanagisako, S. J. (1987). Gender and kinship: Essays toward a unified analysis. Standford: Stanford University Press.

Comas d'Argemir, D. (2014). «La crisis de los cuidados como crisis de reproducción social. Las políticas públicas y más allá». En Periferias, fronteras y diálogos. XIII Congreso de Antropología de la Federación de Asoci- 
aciones de Antropología del Estado Español. Tarragona, Universitat Rovira i Virgili.

-(2017). «El don y la reciprocidad tienen género: Las bases morales de los cuidados». Quaderns-e, 22 (2), 17-32.

Comas d'Argemir, D., Alonso, A. y Deusdad, B. (2018). «Des maris qui soignent leurs épouses âgés. Genre, générations et politiques publiques en Catalogne». Ethnologie Française, 171, 51-64

Comas d'Argemir, D. y Chirinos, C. (2017). «Cuidados no pagados: experiencias y percepciones de los hombres cuidadores en contextos familiares». Revista Murciana de Antropología , 24, 65-86.

Comas D'Argemir, D. y Soronellas, M. (2019). «Men as carers in long-term caring. Doing gender and doing kinship.» Journal of Family Issues, 40 (3), 315-339.

Conlon, C., Timonen, V., Carney, G. y Scharf, T. (2016). «Women (re) negotiating care across family generations. Intersections of gender and socioeconomic status». Gender $\&$ Society, 8 (5), 729-751.

DALY, M. y LEWIS, J. (2000). «The concept of social care and the analysis of contemporary welfare states». British Journal of Sociology, 51, 281-298.

Finch, J. (1989). Family obligations and social change. Cambridge: Polity Press.

Gregorio, C. y Gonzálvez, H. (2012). «Las articulaciones entre género y parentesco en el contexto migratorio: Más allá de la maternidad transnacional». Ankulegi, 16, 43-57.

Hanlon, N. (2012). Masculinities, care and equality: Identity and nurture in men's live. London: Palgrave MacMillan.

$\mathrm{KAY}, \mathrm{R}$. (2013). «'She's like a daughter to me': Insights into care, work and kinship from rural Russia». European Asia Studies, 65, 1136-1153.

Manceron, V. y Segalen, M. (2012). «Entre solidarité résidentielle, sentiments et soutiens publics; les relations de parenté dans la Liberté à Nanterre». Ethnologie Française, XLII (1), 23-36.

MeIL, G. (1997). «La participación masculina en el cuidado de los hijos en la nueva familia urbana española». Papers, 53, 77-99. 
Milligan, C. y Morbey, H. (2016). «Care, coping and identity: Older men's experiences of spousal care-giving». Journal of Ageing Studies, 38, 105114.

Pérez-Orozco, A. (2006). «Amenaza tormenta: La crisis de los cuidados en la reorganización del sistema económico». Revista de Economía Crítica, 5, 7-37.

Pérez-Orozco, A. y López-Gil, S. (2011). Desigualdades a flor de piel. Cadenas globales de cuidados. ONU, Mujeres.

RAZAVI, S. (2007). The political and socialeconomy of care in a development context: Conceptual issues, research questions and policy options. United Nations Research Institute for Social Development. Gender and Development Programme Paper, 3.

Roigé, X. y Soronellas, M. (2018). «Divorce et viellissement. Nouveaux défis sur les rélations d'aide familiale». Ethnologie Française, 171, 465-477. Russell, R. (2001). «In sickness and in health: A qualitative study of elderly men who care for wives with dementia». Journal of Aging Studies, 15, 351-367.

SAHLins, M. (2013). What kinship is-and is not. Chicago, IL: University of Chicago Press.

SARACENO, C. (2010). «Social inequalities in facing old-age dependency: A bigenerational perspective». Journal of European Social Policy, 20, 32-44. Soronellas, M. y Comas d’Argemir, D. (2017). «Hombres cuidadores de personas adultas dependientes. ¿Estrategias ante la crisis o nuevos agentes de cuidado?» En M. R. Herrera y G. Jaraiz (eds.). Pactar el Futuro. Debates para un nuevo consenso en torno al bienestar. Sevilla: Universidad Pablo de Olavide. 